ARTIGO ORIGINAL ORIGINAL ARTICLE

\title{
Sequential biological therapies in metastatic colorectal cancer (mCRC): a cost comparison analysis for wild- type RAS MCRC patients in Brazil
}

\author{
Sequenciamento de biológicos em câncer colorretal \\ metastático (CCRm): uma análise de comparação de \\ custos para pacientes CCRm RAS selvagens no Brasil
}

Rui Fernando Weschenfelder ${ }^{1}$, Carolina Terumi Tsuchiya ${ }^{2}$, Hellen Soo Jin Kim², Jairo Amorim Simões ${ }^{2}$, Cintia Sayuri Kurokawa La Scala²

DOI: $10.21115 / J B E S . v 8 . n 1 . p 24-38$

\section{Keywords:}

metastatic colorectal cancer, bevacizumab, cetuximab, panitumumab, multiple lines, $\mathrm{TML}$

\section{Palavras-chave: \\ câncer colorretal metastático, bevacizumabe, cetuximabe, panitumumabe, múltiplas linhas, TML}

\begin{abstract}
Objective: To compare the treatment costs of different sequences of regimens including monoclonal antibodies in metastatic colorectal cancer ( $\mathrm{CRCm}$ ) treatment for the Brazilian Supplementary Healthcare System. Methods: Sixteen scenarios were analyzed, each one comparing a sequence of bevacizumab TML plus an anti-EGFR therapy in the third-line with another sequence without bevacizumab TML (non-Bev TML) in patients with CRCm wild-type RAS. The anti-EGFRs cetuximab and panitumumab were included. The monthly and total costs of the therapeutic sequences were compared per patient. Results: The sequences with Bev TML were cost-saving in 50\% of all scenarios, and especially observed over regimens starting with cetuximab in the first-line treatment. Regarding scenarios which the non-Bev TML sequences were less costly, they all started with bevacizumab followed by an anti-EGFR biologic drug. Conclusion: The Bev TML regimens were cost-saving compared to scenarios of non-Bev TML which started with cetuximab, and sequential use of bevacizumab beyond progression and the addition of an anti-EGFR biologic drug in the third-line for $\mathrm{mCRC}$ treatment. Considering the remaining scenarios in which Bev-TML was not cost-saving, those starting with Bev presented lower costs in total. Therefore, starting a treatment with bevacizumab seems to enable a more rational management of resource usage, as well as, to allow physicians to add a biologic drug in the third-line, potentially enhancing the long term management of wild-type RAS MCRC.
\end{abstract}

\section{RESUMO}

Objetivo: Comparar o custo de tratamento de diferentes sequências de regimes incluindo anticorpos monoclonais no tratamento de câncer colorretal metastático (CCRm) no Sistema de Saúde Suplementar Brasileiro. Métodos: Dezesseis cenários foram analisados, cada um comparando uma sequência de bevacizumabe TML (BevTML) mais um anti-EGFR em terceira linha, com outra sequência sem bevacizumabe TML (não-Bev TML). Os anti-EGFRs cetuximabe e panitumumabe forma incluídos. Os custos mensais e totais do sequenciamento terapêutico foram comparados por pacientes. Resultados: As sequências com Bev TML trouxeram economia de recursos em 50\% de todos os cenários, e especialmente comparado aos regimes iniciando com cetuximabe em primeira linha de tratamento. Considerando os cenários em que os regimes não-Bev TML apresentaram menos custo, todos iniciaram o sequenciamento com bevacizumabe seguido de um medicamento biológico anti-EGFR. Conclusões: Os regimes Bev TML apresentaram economia de recursos comparado aos cenários com não-Bev TML que iniciaram com cetuximabe, apesar do uso de bevacizumabe em múltiplas linhas e da adição de medicamento biológico anti-EGFR em terceira linha no tratamen-

Recebido em: 12/02/2016. Aprovado para publicação em: 28/03/2016

1. Moinhos de Vento Hospital, Porto Alegre, Brazil;

2. F. Hoffmann-La Roche Ltd, São Paulo, Brazil

Information on financial support: This study was funded by F. Hoffmann-La Roche Ltd.

Corresponding author: Carolina T. Tsuchiya. Roche Brazilian Affiliate Pharmaceuticals Division. Produtos Roche Químicos e Farmacêuticos S.A. Av. Engenheiro Billings, 1729 - Jaguaré - 05321-900 São Paulo-SP Brazil. Office: +55 11 3719-8063. Fax: +55 $113719-7886$. E-mail: carolina.tsuchiya@roche.com 
to de CCRm. Considerando os demais cenários em que os regimes Bev-TML não apresentaram economia de recursos, os regimes iniciando com Bev apresentaram menor custo total. Desta maneira, iniciar o tratamento com bevacizumabe proporciona um gerenciamento mais racional de uso de recursos, assim como, permite aos médicos adicionar um medicamento biológico em terceira linha, potencialmente melhorando o manejo a longo prazo do CCRm com RAS selvagem.

\section{Introduction}

Colorectal cancer (CRC) is a major cause of morbidity and mortality worldwide, with about 1,361,000 new cases and 614,000 deaths in 2012 (GLOBOCAN, 2012). In Brazil, the CRC is the third most common incident of tumors in the population, excluding non-melanoma skin. It is the third most frequent incident in men and second in women. In 2014, it was estimated at around 33 thousand new cases of this disease in the country (INCA, 2014).

In Brazil, the proportion of cases between men and women is $46.2 \%$ and $53.8 \%$ (INCA, 2014). Approximately $25 \%$ of patients present metastatic disease at diagnosis and about $50 \%$ of patients will develop metastatic disease (GLOBOCAN, 2012; Van Cutsem \& Oliveira, 2009).

Over the past 10 years, various combinations of chemotherapy were investigated for the treatment of metastatic CRC (mCRC) (Jackson et al., 2009). Chemotherapy combinations recommended usually contain fluorouracil (5-FU) or capecitabine, associated with oxaliplatin (FOLFOX) or irinotecan (FOLFIRI) (Colucci et al., 2005; Schmoll et al., 2007; Fuchs et al., 2007; Van Cutsem et al., 2001; de Gramont et al., 2000; Douillard et al., 2000; Saltz et al., 2000).

Recently, molecular targeted therapies have had special attention from the medical community worldwide. Especially in the treatment of mCRC, bevacizumab (Bev), a humanized monoclonal antibody that targets the vascular endothelial growth factor (VEGF), has shown favorable results when combined with chemotherapy regimens in phase III studies (Hurwitz et al., 2004; Welch et al., 2010; Guan et al., 2011; Hurwitz et al., 2005). Cetuximab (Cet) and panitumumab (Pan), monoclonal antibodies that target the epidermal growth factor receptor (EGFR), also demonstrated favorable results in combination with chemotherapy in the treatment of $\mathrm{mCRC}$, but their benefits are restricted to patients with wild-type RAS (wtRAS) (Bokemeyer et al., 2012; Sobrero et al., 2008; Peeters et al., 2010), while Bev can be included as a treatment option for $\mathrm{mCRC}$ patients regardless of their RAS status (Fuchs et al., 2007; Hurwitz et al., 2004; Saltz et al., 2008). Therapy combining these agents is the most commonly used regimen for $\mathrm{mCRC}$ in Brazil.

The best sequence of use of these therapies, particularly regarding biological or molecular targeted therapies in mCRC, is still undefined (Bennouna et al., 2013). In 2012, ML18147 trial (Bennouna et al., 2013) - a randomized, open, multicenter study - was published. This study assessed the continuous use of Bev associated with chemotherapy (FOLFOX, FOLFIRI) in second-line (2L) treatment in patients who were refractory to chemotherapy with Bev in first-line (1L) therapy. The study showed higher overall survival rates in patients treated with Bev associated with chemotherapy through multiple lines (TML) in $\mathrm{mCRC}$.

With a similar approach, in 2014, Rautenberg et al. evaluated clinical and economic outcomes of sequences with Bev in $1 \mathrm{~L}$ and $2 \mathrm{~L}$, followed by an anti-EGFR in the third-line (3L). The authors concluded that Bev TML followed by $3 \mathrm{~L}$ anti-EGFR could potentially yield better health outcomes, associated with saving costs when compared to $1 \mathrm{~L}$ anti-EGFR regimens.

The aim of this study was to compare treatments of different sequences of therapy that include Bev, Cet or Pan for the treatment of $\mathrm{mCRC}$ from a payer perspective in the Brazilian Supplementary Healthcare System. None other biologic drug is available in Brazil for mCRC treatment.

\section{Methods}

The treatment sequences described in the ML18147 trial (Bennouna et al., 2013) were compared to commonly used regimens to treat $\mathrm{mCRC}$, according to main Phase III randomized clinical trials summarized in Table 1 and the local products labels.

In order to compare the economic outcomes, the monthly cost and the total cost of the sequence per patient, according to the $1 \mathrm{~L}, 2 \mathrm{~L}$ and $3 \mathrm{~L}$ combinations, calculations were made using a tool developed in Microsoft Excel ${ }^{\circledast}$ (Microsoft, Redmond, WA), which allowed estimating the costs associated with the treatment of $\mathrm{mCRC}$.

The focus of the analysis was the direct costs of treatment, including drug acquisition, intravenous (IV) administration fees and supportive care in the private healthcare system. Neither indirect costs, nor the costs of managing adverse events were included in the calculations.

\section{Scenarios}

To estimate the treatment costs of the use of Bev or Cet/Pan sequences, 16 different comparison scenarios were developed (Table 2). These drug regimens are the most widely used treatment for $\mathrm{mCRC}$ in $1 \mathrm{~L}$ and $2 \mathrm{~L}$ in Brazil, and they are described in most treatment guidelines for metastatic disease (Van Cutsem \& Oliveira, 2009; Sobrero et al., 2008; Peeters et al., 2010; Rautenberg et al., 2014; Van Cutsem et al., 2007; Chari et al., 2006; Glimelius \& Oliveira, 2008; Glimelius \& Oliveira, 2009; Nordlinger et al., 2009; Nordlinger et al., 2007; Papamichael et al., 2009; Van Cutsem \& Oliveira, 2008). 
Table 1. Studies referenced for bevacizumab and cetuximab

\begin{tabular}{|c|c|c|c|}
\hline Study name & Study reference & Regimen & Outcomes \\
\hline \multicolumn{4}{|l|}{ Bevacizumab } \\
\hline N016966 & Saltz et al., 2008 & $\begin{array}{l}\text { Bev } 5 \mathrm{mg} / \mathrm{kg}+\text { FOLFOX4 } \\
\text { vs. } \\
\text { FOLFOX4 }\end{array}$ & $\begin{array}{l}\text { Primary Endpoint: mPFS - } 9.4 \text { mo vs. } 8.0 \text { mo } \\
\text { (HR, 0.83; } 97.5 \% \mathrm{Cl}, 0.72 \text { to } 0.95 ; \mathrm{p}=0.0023) \\
\text { Secondary Endpoint: } \mathrm{mOS}-21.3 \text { mo vs. } 19.9 \mathrm{mo} \\
(\mathrm{HR}, 0.89 ; 97.5 \% \mathrm{Cl}, 0.76 \text { to } 1.03 ; p=0.077)\end{array}$ \\
\hline ML18147 & Bennouna et al., 2013 & $\begin{array}{l}\text { Bev } 5 \text { mg/kg + simplified FOLFIRI/ } \\
\text { Bev } 5 \text { mg/kg + FOLFOX } 6 \\
\text { vs. } \\
\text { simplified FOLFIRI/ FOLFOX }\end{array}$ & $\begin{array}{l}\text { Primary Endpoint: mOS - } 11.2 \text { mo vs. } 9.8 \\
\text { mo (hazard ratio 0.81, } 95 \% \text { Cl 0.69-0.94; } \\
\text { unstratified log-rank test } p=0.0062 \text { ) } \\
\text { Secondary Endpoint: mPFS - } 5.7 \text { mo vs. } 4.1 \text { mo (HR } \\
0.68,95 \% \text { Cl 0.59-0.78; unstratified log-rank } p<0.0001)\end{array}$ \\
\hline E3200 & Giantonio et al., 2007 & $\begin{array}{l}\text { Bev } 10 \mathrm{mg} / \mathrm{kg}+\text { FOLFOX4 } \\
\text { vs. } \\
\text { FOLFOX4 }\end{array}$ & $\begin{array}{l}\text { Primary Endpoint: mOS }-12.9 \text { mo vs. } \\
10.8 \text { mo }(H R=0.75 ; p=0.0011) \\
\text { Secondary Endpoint: } m P F S-7.3 \text { mo } \\
\text { vs. } 4.7 \text { mo }(H R=0.61 ; p<0.0001)\end{array}$ \\
\hline BICC-C & Fuchs et al., 2007 & $\begin{array}{l}\text { Bev } 5 \mathrm{mg} / \mathrm{kg}+\text { FOLFIRI } \\
\text { vs. } \\
\text { Bev } 7.5 \mathrm{mg} / \mathrm{kg}+\mathrm{mlFL}\end{array}$ & $\begin{array}{l}\text { Primary Endpoint: mPFS - } 11.2 \text { mo vs. } 8.3 \text { mo ( } p=0.28 \text { ) } \\
\text { Secondary Endpoint: mOS - } 28.0 \text { mo vs. } 19.2 \text { mo } \\
\text { (HR for death }=1.79 ; 95 \% \mathrm{Cl}, 1.12-2.88 ; p=0.037 \text { ) }\end{array}$ \\
\hline CALGB80405 & Venook et al., 2014 & $\begin{array}{l}\text { Bev } 5 \mathrm{mg} / \mathrm{kg}+\text { mFOLFOX6/ } \\
\text { Bev } 5 \mathrm{mg} / \mathrm{kg}+\text { FOLFIRI } \\
\text { vs. } \\
\text { Cet } 400 \mathrm{mg} / \mathrm{m}^{2} 1 \text { st cycle, then } \\
250 \mathrm{mg} / \mathrm{m}^{2} \text { weekly + mFOLFOX6/ } \\
\text { Cet } 400 \mathrm{mg} / \mathrm{m}^{2} 1 \text { st cycle, then } \\
250 \mathrm{mg} / \mathrm{m}^{2} \text { weekly + FOLFIRI }\end{array}$ & $\begin{array}{l}\text { Primary Endpoint: mOS - } 29.04 \text { mo vs. } 29.93 \\
\text { mo (HR=0.92, 95\% CI 0.78, 1.09; } p=0.34) \\
\text { Secondary Endpoint: mPFS - } 10.84 \text { mo vs. } 10.45 \\
\text { mo (statistical analysis not published) }\end{array}$ \\
\hline \multicolumn{4}{|c|}{ Cetuximab and BSC } \\
\hline CRYSTAL & Van Cutsem et al., 2009 & $\begin{array}{l}\text { Cet } 400 \mathrm{mg} / \mathrm{m}^{2} \text { 1st cycle, then } \\
250 \mathrm{mg} / \mathrm{m}^{2} \text { weekly + FOLFIRI } \\
\text { vs. } \\
\text { FOLFIRI }\end{array}$ & $\begin{array}{l}\text { Primary Endpoint: } \mathrm{mPFS}-8.9 \text { mo vs. } 8.0 \text { mo } \\
(\mathrm{HR}=0.85 ; 95 \% \mathrm{Cl}, 0.72 \text { to } 0.99 ; \mathrm{p}=0.048) \\
\text { Secondary Endpoint: } \mathrm{mOS}-19.9 \text { mo vs. } 18.6 \\
\mathrm{mo}(\mathrm{HR}=0.93 ; 95 \% \mathrm{Cl}, 0.81 \text { to } 1.07 ; \mathrm{p}=0.31)\end{array}$ \\
\hline EPIC & Sobrero et al., 2008 & $\begin{array}{l}\text { Cet } 400 \mathrm{mg} / \mathrm{m}^{2} \text { 1st cycle, then } \\
250 \mathrm{mg} / \mathrm{m}^{2} \text { weekly }+\mid \mathrm{RI} \\
\text { Vs. } \\
\text { IRI }\end{array}$ & $\begin{array}{l}\text { Primary Endpoint: } \mathrm{mOS}-10.7 \text { mo vs. } 10.0 \text { mo } \\
\text { (HR=0.975; } 95 \% \mathrm{Cl}, 0.854 \text { to } 1.114 ; \mathrm{p}=0.71 \text { ) } \\
\text { Secondary Endpoint: mPFS }-4.0 \text { mo vs. } 2.6 \mathrm{mo} \\
\text { (HR=0.692; } 95 \% \mathrm{Cl}, 0.617 \text { to } 0.776 ; \mathrm{p}<0.0001)\end{array}$ \\
\hline Co.17 & Karapetis et al., 2008 & $\begin{array}{l}\text { Cet } 400 \mathrm{mg} / \mathrm{m}^{2} \text { 1st cycle, then } \\
250 \mathrm{mg} / \mathrm{m}^{2} \text { weekly }+ \text { BSC } \\
\text { vs. } \\
\text { BSC }\end{array}$ & $\begin{array}{l}\text { Primary Endpoint: } \mathrm{mOS}-9.5 \text { mo vs. } 4.8 \mathrm{mo} \\
\text { (HR=0.55; } 95 \% \mathrm{Cl}, 0.41 \text { to } 0.74 ; \mathrm{p}<0.001) \\
\text { Secondary Endpoint: } \mathrm{mPFS}-3.7 \text { mo vs. } 1.9 \mathrm{mo} \\
\text { (HR=0.40; } 95 \% \mathrm{Cl}, 0.30 \text { to } 0.54 ; \mathrm{p}<0.001)\end{array}$ \\
\hline \multicolumn{4}{|l|}{ Panitumumab } \\
\hline Peeters & Peeters et al., 2010 & $\begin{array}{l}\text { Pan } 6 \mathrm{mg} / \mathrm{kg}+\text { FOLFIRI } \\
\text { vs. } \\
\text { FOLFIRI }\end{array}$ & $\begin{array}{l}\text { Coprimary Endpoints: mPFS }-5.9 \text { mo vs. } 3.9 \text { mo } \\
(\mathrm{HR}=0.73 ; 95 \% \mathrm{Cl}, 0.59 \text { to } 0.90 ; \mathrm{p}=0.004) ; \mathrm{mOS}-14.5 \\
\text { mo vs. } 12.5 \mathrm{mo}(\mathrm{HR}=0.85,95 \% \mathrm{Cl}, 0.70 \text { to } 1.04 ; \mathrm{p}=0.12)\end{array}$ \\
\hline Van Cutsem & Van Cutsem et al., 2007 & $\begin{array}{l}\text { Pan } 6 \mathrm{mg} / \mathrm{kg}+\mathrm{BSC} \\
\text { vs. } \\
\text { BSC }\end{array}$ & $\begin{array}{l}\text { Primary Endpoint: } \mathrm{mPFS}-8.0 \text { weeks vs. } 7.3 \text { weeks } \\
\text { (HR=0.54; } 95 \% \mathrm{Cl}, 0.44 \text { to } 0.66, \mathrm{p}<0.0001) \\
\text { Secondary Endpoint: } \mathrm{mOS}-\mathrm{HR}=1.00 ; 95 \% \mathrm{Cl}, 0.82 \text { to } 1.22\end{array}$ \\
\hline
\end{tabular}

*Bev: bevacizumab; Cet: cetuximab; Pan: panitumumab; BSC: best supportive care; mPFS: median progression free survival; mOS: median overall survival; HR: hazard ratio

\section{Treatment duration}

Two different approaches were used to estimate the treatment duration. Each scenario was evaluated based on median progression-free survival (PFS) data from randomized clinical trials and also on the estimated average treatment duration for each line from Rautenberg et al (2014). Data on treatment duration are described in Table 3.

\section{Costs}

The acquisition costs for each biological and chemotherapeutic drug included in the analysis were obtained from local official databases. Acquisition costs per milligram of each drug are shown in Table 4.

Supportive care and intravenous administration costs were obtained from Brazilian Hierarchical Classification of 
Table 2. Treatment regimens compared in the cost analysis

\begin{tabular}{|c|c|c|c|}
\hline & $\begin{array}{l}\text { Bevacizumab } \\
\text { Multiple Line }\end{array}$ & Non-Bevacizumab Multiple Lines & \\
\hline \multirow[t]{3}{*}{ Scenario 1} & $1 \mathrm{~L}$ & Bev + FOLFOX4 (NO16966) & Cet + FOLFIRI (CRYSTAL) \\
\hline & $2 \mathrm{~L}$ & Bev + simplified FOLFIRI (ML18147) & Bev + FOLFOX4 (E3200) \\
\hline & $3 \mathrm{~L}$ & Cet + BSC (CO.17) & $\mathrm{BSC}(\mathrm{CO} .17)$ \\
\hline \multirow[t]{3}{*}{ Scenario 2} & $1 \mathrm{~L}$ & $\mathrm{Bev}+\mathrm{FOLFIRI}(\mathrm{BICC}-\mathrm{C})$ & Cet + FOLFIRI (CRYSTAL) \\
\hline & $2 \mathrm{~L}$ & Bev + FOLFOX6 (ML18147) & Bev + FOLFOX4 (E3200) \\
\hline & $3 \mathrm{~L}$ & Cet + BSC (CO.17) & $\mathrm{BSC}(\mathrm{CO} .17)$ \\
\hline \multirow[t]{3}{*}{ Scenario 3} & $1 \mathrm{~L}$ & Bev + mFOLFOX6 (CALGB) & Cet + FOLFIRI (CRYSTAL) \\
\hline & $2 \mathrm{~L}$ & Bev + simplified FOLFIRI (ML18147) & Bev + FOLFOX4 (E3200) \\
\hline & $3 L$ & Cet + BSC (CO.17) & BSC (CO.17) \\
\hline \multirow[t]{3}{*}{ Scenario 4} & $1 \mathrm{~L}$ & Bev + FOLFIRI (CALGB) & Cet + FOLFIRI (CRYSTAL) \\
\hline & $2 \mathrm{~L}$ & Bev + FOLFOX6 (ML18147) & Bev + FOLFOX4 (E3200) \\
\hline & $3 \mathrm{~L}$ & Cet + BSC (CO.17) & $\mathrm{BSC}(\mathrm{CO} .17)$ \\
\hline \multirow[t]{3}{*}{ Scenario 5} & $1 \mathrm{~L}$ & Bev+ FOLFOX4 (NO16966) & Bev + FOLFOX 4 (NO16966) \\
\hline & $2 \mathrm{~L}$ & Bev + simplified FOLFIRI (ML18147) & Pan + FOLFIRI (Peeters) \\
\hline & $3 \mathrm{~L}$ & Cet + BSC (CO.17) & BSC (CO.17) \\
\hline \multirow[t]{3}{*}{ Scenario 6} & $1 \mathrm{~L}$ & $\mathrm{Bev}+\mathrm{FOLFIRI}(\mathrm{BICC}-\mathrm{C})$ & Bev + FOLFOX 4 (NO16966) \\
\hline & $2 \mathrm{~L}$ & Bev + FOLFOX6 (ML18147) & Pan + FOLFIRI (Peeters) \\
\hline & $3 \mathrm{~L}$ & Cet + BSC (CO.17) & $\mathrm{BSC}(\mathrm{CO} .17)$ \\
\hline \multirow[t]{3}{*}{ Scenario 7} & $1 \mathrm{~L}$ & Bev + FOLFOX4 (NO16966) & Bev + FOLFOX 4 (NO16966) \\
\hline & $2 \mathrm{~L}$ & Bev + simplified FOLFIRI (ML18147) & Pan + FOLFIRI (Peeters) \\
\hline & $3 L$ & Pan + BSC (Van Cutsem) & $\mathrm{BSC}(\mathrm{CO} .17)$ \\
\hline \multirow[t]{3}{*}{ Scenario 8} & $1 \mathrm{~L}$ & Bev+ FOLFIRI (BICC-C) & Bev + FOLFOX 4 (NO16966) \\
\hline & $2 \mathrm{~L}$ & Bev + FOLFOX6 (ML18147) & Pan + FOLFIRI (Peeters) \\
\hline & $3 \mathrm{~L}$ & Pan + BSC (Van Cutsem) & $\mathrm{BSC}(\mathrm{CO} .17)$ \\
\hline \multirow[t]{3}{*}{ Scenario 9} & $1 \mathrm{~L}$ & Bev + FOLFOX4 (NO16966) & Bev + FOLFOX 4 (NO16966) \\
\hline & $2 \mathrm{~L}$ & Bev + simplified FOLFIRI (ML18147) & Cet + IRI (EPIC) \\
\hline & $3 \mathrm{~L}$ & Cet + BSC (CO.17) & $\mathrm{BSC}(\mathrm{CO} .17)$ \\
\hline \multirow[t]{3}{*}{ Scenario 10} & $1 \mathrm{~L}$ & Bev + FOLFIRI (BICC-C) & Bev + FOLFOX 4 (NO16966) \\
\hline & $2 \mathrm{~L}$ & Bev + FOLFOX6 (ML18147) & Cet + IRI (EPIC) \\
\hline & $3 \mathrm{~L}$ & Cet + BSC (CO.17) & BSC (CO.17) \\
\hline \multirow[t]{3}{*}{ Scenario 11} & $1 \mathrm{~L}$ & Bev + FOLFOX4 (NO16966) & Bev + FOLFOX 4 (NO16966) \\
\hline & $2 \mathrm{~L}$ & Bev + simplified FOLFIRI (ML18147) & Cet + IRI (EPIC) \\
\hline & $3 \mathrm{~L}$ & Pan + BSC (Van Cutsem) & $\mathrm{BSC}(\mathrm{CO} .17)$ \\
\hline \multirow[t]{3}{*}{ Scenario 12} & $1 \mathrm{~L}$ & Bev + FOLFIRI (BICC-C) & Bev + FOLFOX 4 (NO16966) \\
\hline & $2 \mathrm{~L}$ & Bev + FOLFOX6 (ML18147) & Cet + IRI (EPIC) \\
\hline & $3 \mathrm{~L}$ & Pan + BSC (Van Cutsem) & $\mathrm{BSC}(\mathrm{CO} .17)$ \\
\hline \multirow[t]{3}{*}{ Scenario 13} & $1 \mathrm{~L}$ & Bev + FOLFOX4 (NO16966) & Cet + FOLFIRI (CRYSTAL) \\
\hline & $2 \mathrm{~L}$ & Bev + simplified FOLFIRI (ML18147) & Bev + FOLFOX4 (E3200) \\
\hline & $3 L$ & Pan + BSC (Van Cutsem) & $\mathrm{BSC}(\mathrm{CO} .17)$ \\
\hline \multirow[t]{3}{*}{ Scenario 14} & $1 \mathrm{~L}$ & Bev + FOLFIRI (BICC-C) & Cet + FOLFIRI (CRYSTAL) \\
\hline & $2 \mathrm{~L}$ & Bev + FOLFOX6 (ML18147) & Bev + FOLFOX4 (E3200) \\
\hline & $3 \mathrm{~L}$ & Pan + BSC (Van Cutsem) & $\mathrm{BSC}(\mathrm{CO} .17)$ \\
\hline \multirow[t]{3}{*}{ Scenario 15} & $1 \mathrm{~L}$ & Bev + mFOLFOX6 (CALGB) & Cet + FOLFIRI (CRYSTAL) \\
\hline & $2 \mathrm{~L}$ & Bev + simplified FOLFIRI (ML18147) & Bev + FOLFOX4 (E3200) \\
\hline & $3 \mathrm{~L}$ & Pan + BSC (Van Cutsem) & $\mathrm{BSC}(\mathrm{CO} .17)$ \\
\hline \multirow[t]{3}{*}{ Scenario 16} & $1 \mathrm{~L}$ & Bev + FOLFIRI (CALGB) & Cet + FOLFIRI (CRYSTAL) \\
\hline & $2 \mathrm{~L}$ & Bev + FOLFOX6 (ML18147) & Bev + FOLFOX4 (E3200) \\
\hline & $3 L$ & Pan + BSC (Van Cutsem) & BSC (CO.17) \\
\hline
\end{tabular}

*FOLFOX: bolus and infusion fluorouracil/ leucovorin + oxaliplatin; FOLFIRI: bolus and infusion fluorouracil/ leucovorin + irinotecan; Bev: bevacizumab; Cet: cetuximab; Pan: panitumumab 
Table 3. Estimated treatment time based on standard treatment time and PFS reported on clinical trials

\begin{tabular}{|c|c|c|c|c|}
\hline Treatment Line & Study Name & Regimen & PFS (months) & $\begin{array}{l}\text { Average treatment } \\
\text { duration* }\end{array}$ \\
\hline $1 \mathrm{~L}$ & N016966 & Bev 5 mg/kg+FOLFOX4 & 9.4 & 6.1 \\
\hline $1 \mathrm{~L}$ & $\mathrm{BICC}-\mathrm{C}$ & Bev 5 mg/kg+FOLFIRI & 11.2 & 6.1 \\
\hline $1 \mathrm{~L}$ & CALGB80405 & Bev 5 mg/kg+mFOLFOX6 & 10.8 & 6.1 \\
\hline $1 \mathrm{~L}$ & CALGB80405 & Bev 5 mg/kg+FOLFIRI & 10.8 & 6.1 \\
\hline $1 \mathrm{~L}$ & CRYSTAL & Cet $250 \mathrm{mg} / \mathrm{m}^{2}+\mathrm{FOLFIRI}$ & 9.9 & 6.1 \\
\hline $1 \mathrm{~L}$ & CALGB80405 & Cet $250 \mathrm{mg} / \mathrm{m}^{2}+\mathrm{FOLFIRI}$ & 10.4 & 6.1 \\
\hline $2 \mathrm{~L}$ & E3200 & Bev $10 \mathrm{mg} / \mathrm{kg}+\mathrm{FOLFOX} 4$ & 7.3 & 4.0 \\
\hline $2 \mathrm{~L}$ & ML18147 & Bev 5 mg/kg+ simplified FOLFIRI & 5.7 & 4.0 \\
\hline $2 \mathrm{~L}$ & ML18147 & Bev $5 \mathrm{mg} / \mathrm{kg}+\mathrm{FOLFOX6}$ & 5.7 & 4.0 \\
\hline $2 \mathrm{~L}$ & EPIC & Cet $250 \mathrm{mg} / \mathrm{m}^{2}+\mid \mathrm{RI}$ & 4.0 & 4.0 \\
\hline $2 \mathrm{~L}$ & Peeters & Pan 6 mg/kg+ FOLFIRI & 5.9 & 4.0 \\
\hline $3 \mathrm{~L}$ & CO.17 & Cet $250 \mathrm{mg} / \mathrm{m}^{2}+\mathrm{BSC}$ & 3.7 & 2.7 \\
\hline $3 \mathrm{~L}$ & CO.17 & $\mathrm{BSC}$ & 1.9 & 2.7 \\
\hline $3 L$ & Van Cutsem & Pan $6 \mathrm{mg} / \mathrm{kg}+\mathrm{BSC}$ & 1.9 & 2.7 \\
\hline
\end{tabular}

*Rautenberg et al. tFOLFOX: bolus and infusion fluorouracil/ leucovorin + oxaliplatin; FOLFIRI: bolus and infusion fluorouracil/ leucovorin + irinotecan; Bev: bevacizumab; Cet: cetuximab; Pan: panitumumab

Table 4. Description of costs per mg concerning biological and chemotherapeutic treatments evaluated in the study

\begin{tabular}{lc}
\hline Biological treatment & Cost per $\mathbf{m g}(\mathbf{R} \$)^{*}$ \\
\hline Bevacizumab & 13.59 \\
\hline Cetuximab & 7.71 \\
\hline Panitumumab & 12.86 \\
\hline Chemotherapeutic treatment & Cost per $\mathbf{m g}(\mathbf{R} \$)^{*}$ \\
\hline 5-Fluorouracil (5-FU) & 0.04 \\
\hline Oxaliplatin & 38.26 \\
\hline Irinotecan & 16.57 \\
\hline Folinic acid & 1.01 \\
\hline
\end{tabular}

*Source: CMED (April 2015) - Ex-Factory price, ICMS 18\%

Medical Procedures (CBHPM) table from the Brazilian Medical Association and are described in Tables 5 and 6 .

In the case of individual drugs with the same duration of the cycle, it was considered that they are administered sequentially with the cost of a single administration.

\section{Results}

The results presented in Tables 7-24 refer to the average cost for treatment of a single patient, considering the amounts described in the "Costs" section that comprises each treatment regimen.

To confirm the robustness of the data, a one-way sensitivity analysis was performed to measure the impact of each parameter and assess the uncertainty of the results. The para- meters described as shown in Table 25 varied in a 10\% range (up and down). A sensitivity analysis within the same conditions was conducted for all scenarios.

A one-way analysis was performed and revealed that, in all scenarios, the maximum variation was $6 \%$ and occurred varying the biologic drug cost acquisition, weight and $1 \mathrm{~L}$ treatment (months). All simulations showed that Bev TML sequences were more influenced by the patients' body surface area (BSA) and weight, Bev acquisition price and months of treatment ( $1 \mathrm{~L}$ and $2 \mathrm{~L}$ treatments). For Non-TML regimens, it was observed that besides those parameters, the total cost treatment was influenced by cetuximab and panitumumab acquisition prices.

\section{Discussion}

According to the results of the ML18147 trial (Bennouna et al., 2013), Bev resistance seems to occur at different times and with different mechanisms than chemotherapy resis-

Table 5. Supportive care and intravenous administration

\begin{tabular}{lcc}
\hline Procedure & Cost (R\$) & Period \\
\hline Best supportive care* $^{*}$ & 255.74 & Monthly \\
\hline $\begin{array}{l}\text { Administration of } \\
\text { intravenous therapy }\end{array}$ & 184.38 & Per adm \\
\hline Administration of infusion therapy & 251.49 & $\begin{array}{c}\text { Infusion } \\
(\geq 22 \mathrm{~h})\end{array}$ \\
\hline
\end{tabular}

*see Table 6 for further information 
Table 6. Supportive care costs (per month)

\begin{tabular}{|c|c|c|c|}
\hline Procedure & Quantity & $\begin{array}{l}\text { Cost per } \\
\text { unit (R\$)* }\end{array}$ & $\begin{array}{l}\text { Cost per } \\
\text { month (R\$) }\end{array}$ \\
\hline Paliative Radiotherapy & 0.17 & 82.11 & 13.69 \\
\hline $\begin{array}{l}\text { Physical examination } \\
\text { and history (medical } \\
\text { consultation) }\end{array}$ & 0.33 & 65.45 & 21.82 \\
\hline Morphine 10 mg (tablet) & 90.00 & 0.47 & 42.21 \\
\hline Osteolysis inhibitor & 1.00 & $1^{\prime} 089.30$ & 108.93 \\
\hline $\begin{array}{l}\text { Radiograph (bone } \\
\text { metastasis control) }\end{array}$ & 0.25 & 308.94 & 7.72 \\
\hline $\begin{array}{l}\text { Radiograph (lung } \\
\text { metastasis control) }\end{array}$ & 0.25 & 35.13 & 1.32 \\
\hline $\begin{array}{l}\text { Abdominal computarized } \\
\text { tomography }\end{array}$ & 0.17 & 620.38 & 31.02 \\
\hline $\begin{array}{l}\text { Complete blood } \\
\text { count }(C B C)\end{array}$ & 0.33 & 12.34 & 4.11 \\
\hline Glucose dosage & 0.33 & 5.56 & 1.85 \\
\hline Urea dosage & 0.33 & 5.56 & 1.85 \\
\hline Creatinine dosage & 0.33 & 5.56 & 1.85 \\
\hline $\begin{array}{l}\text { Alkaline phosphatase } \\
\text { dosage }\end{array}$ & 0.33 & 10.24 & 3.41 \\
\hline GT gamma & 0.33 & 10.24 & 3.41 \\
\hline $\begin{array}{l}\text { Glutamic oxalacetic } \\
\text { transminase dosage }\end{array}$ & 0.33 & 10.24 & 3.41 \\
\hline $\begin{array}{l}\text { Glutamic pyruvate } \\
\text { transminase dosage }\end{array}$ & 0.33 & 10.69 & 3.56 \\
\hline Sodium dosage & 0.33 & 5.56 & 1.85 \\
\hline Potassium dosage & 0.33 & 5.56 & 1.85 \\
\hline Calcium dosage & 0.33 & 5.56 & 1.85 \\
\hline TOTAL (R\$) & 255.74 & & \\
\hline
\end{tabular}

*Brazilian Hierarchical Classification of Medical Procedures (CBHPM), 2014 tance, suggesting that Bev remains effective after the development of resistance to chemotherapy. Therefore, the factors that could help define the best sequence of treatment - such as the expression of wtRAS; regimens with higher response rates to turn initially unresectable lesions into resectable lesions; performance status; and acceptable toxicities of patients - should be considered (Cartwright, 2012). Also, based on the ML18147 trial, it is possible to assume that the use of Bev beyond progression can also be studied in other tumors in other parts of the body such as the lung and breast.

Two randomized phase III clinical trials aimed to compare the efficacy of Bev or Cet in first-line treatment of mCRC, FIRE-3 (Heinemann et al., 2014) and CALGB 80405 (Venook et al., 2014). In FIRE-3, patients were randomized to receive FOLFIRI plus Cet or FOLFIRI plus Bev with a primary endpoint of response rate according to RECIST. In the intent-to-treat (ITT) population (KRAS exon 2 wild type), there was no significant difference in the primary endpoint between treatment arms (odds ratio 1.18; $\mathrm{p}=0.18$ ). Progression-free survival (PFS) was also similar between both groups (hazard ratio [HR] 1.06; $p=0.55)$, however, overall survival (OS), a secondary endpoint, was better in the Cet arm (hazard ratio [HR], 0.77; $p=0.017$ ). This finding is still to be explained, although the Bev group in this study seems to have an outlier result of OS survival when compared to other studies (CALGB 80405 (Venook et al., 2014), TRIBE (Cremolini et al., 2015); hypothesizing that this arm could have received less efficacy treatments in subsequent lines.

The results from the CALGB 80405 study (Venook et al., 2014) were presented as well in 2014, demonstrating similar efficacy outcomes (overall survival and progression-free survival) of Bev and Cet combined with chemotherapy ( $\mathrm{mFOL-}$ FOX6 or FOLFIRI) in the first-line treatment of $\mathrm{mCRC}$ in patients with wild-type KRAS. The quality of life was assessed at baseli-

Table 7. Total costs for each treatment regimen - Scenario 1

\begin{tabular}{|c|c|c|c|c|c|}
\hline & Treatment Regimen & $\begin{array}{c}\text { Treatment } \\
\text { months (PFS) }\end{array}$ & $\begin{array}{l}\text { Total Cost } \\
\text { (R\$) }\end{array}$ & $\begin{array}{l}\text { Treatment } \\
\text { months* }\end{array}$ & $\begin{array}{l}\text { Total Cost } \\
\text { (R\$) }\end{array}$ \\
\hline \multicolumn{6}{|c|}{ Bevacizumab TML } \\
\hline $1 \mathrm{~L}$ & Bev + FOLFOX4 (NO16966) & 9.4 & $226^{\prime} 411.94$ & 6.1 & $146^{\prime} 926.89$ \\
\hline $2 \mathrm{~L}$ & Bev + simplified FOLFIRI (ML18147) & 5.7 & $137^{\prime} 549.62$ & 4.0 & $96^{\prime} 526.05$ \\
\hline $3 L$ & CET + BSC - wild-type KRAS (CO.17) & 3.7 & $59^{\prime} 866.50$ & 2.7 & $44^{\prime} 230.45$ \\
\hline Total & & & $423^{\prime} 828.06$ & & $287^{\prime} 683.40$ \\
\hline \multicolumn{6}{|c|}{ Non-bevacizumab TML } \\
\hline $1 \mathrm{~L}$ & Cet + FOLFIRI (CRYSTAL) & 9.9 & $291^{\prime} 047.28$ & 6.1 & $180^{\prime} 104.87$ \\
\hline $2 \mathrm{~L}$ & Bev 10 mg + FOLFOX (E3200) & 7.3 & $248^{\prime} 216.37$ & 4.0 & $136^{\prime} 008.97$ \\
\hline $3 \mathrm{~L}$ & BSC - wild-type KRAS (CO.17) & 1.9 & 485.90 & 2.7 & 690.49 \\
\hline Total & & & $5399^{\prime} 479.55$ & & $316^{\prime} 804.34$ \\
\hline
\end{tabular}


Table 8. Total costs for each treatment regimen - Scenario 2

\begin{tabular}{|c|c|c|c|c|c|}
\hline & Treatment Regimen & $\begin{array}{c}\text { Treatment } \\
\text { months (PFS) }\end{array}$ & Total Cost (R\$) & $\begin{array}{c}\text { Treatment } \\
\text { months* }\end{array}$ & Total Cost (R\$) \\
\hline \multicolumn{6}{|c|}{ Bevacizumab TML } \\
\hline $1 \mathrm{~L}$ & $\mathrm{Bev}+\mathrm{FOLFIRI}(\mathrm{BICC}-\mathrm{C})$ & 11.2 & $270^{\prime} 272.94$ & 6.1 & $147^{\prime} 202.22$ \\
\hline $2 \mathrm{~L}$ & Bev + FOLFOX (ML18147) & 5.7 & $155^{\prime} 706.86$ & 4.0 & $109^{\prime} 267.97$ \\
\hline $3 \mathrm{~L}$ & CET + BSC - wild-type KRAS (CO.17) & 3.7 & $59^{\prime} 866.50$ & 2.7 & $44^{\prime} 230.45$ \\
\hline Total & & & $485^{\prime} 846.29$ & & $300^{\prime} 700.65$ \\
\hline \multicolumn{6}{|c|}{ Non-bevacizumab TML } \\
\hline $1 \mathrm{~L}$ & Cet + FOLFIRI (CRYSTAL) & 9.9 & $291^{\prime} 047.28$ & 6.1 & $180^{\prime} 104.87$ \\
\hline $2 \mathrm{~L}$ & Bev + FOLFOX 4 (E3200) & 7.3 & $248^{\prime} 216.37$ & 4.0 & $136^{\prime} 008.97$ \\
\hline $3 \mathrm{~L}$ & BSC - wild-type KRAS (CO.17) & 1.9 & 485.90 & 2.7 & 690.49 \\
\hline Total & & & $539^{\prime} 479.55$ & & $316^{\prime} 804.34$ \\
\hline
\end{tabular}

*Standard treatment duration; PFS: progression-free survival; TML: through multiple lines; Bev: bevacizumab; Cet: cetuximab; BSC: best supportive care

Table 9. Total costs for each treatment regimen - Scenario 3

\begin{tabular}{|c|c|c|c|c|c|}
\hline & Treatment Regimen & $\begin{array}{c}\text { Treatment } \\
\text { months (PFS) }\end{array}$ & Total Cost (R\$) & $\begin{array}{l}\text { Treatment } \\
\text { months* }\end{array}$ & Total Cost (R\$) \\
\hline \multicolumn{6}{|c|}{ Bevacizumab TML } \\
\hline $1 \mathrm{~L}$ & Bev + mFOLFOX6 (CALGB/SWOG 80405) & 10.8 & $271^{\prime} 592.65$ & 6.1 & $153^{\prime} 399.55$ \\
\hline $2 \mathrm{~L}$ & Bev + simplified FOLFIRI (ML18147) & 5.7 & $137^{\prime} 549.62$ & 4.0 & $96^{\prime} 526.05$ \\
\hline $3 \mathrm{~L}$ & CET + BSC - wild-type KRAS (CO.17) & 3.7 & $59^{\prime} 866.50$ & 2.7 & $44^{\prime} 230.45$ \\
\hline Total & & & $469^{\prime} 008.78$ & & $294^{\prime} 156.06$ \\
\hline \multicolumn{6}{|c|}{ Non-bevacizumab TML } \\
\hline $1 \mathrm{~L}$ & Cet + FOLFIRI (CALGB/SWOG 80405) & 10.4 & $305^{\prime} 644.96$ & 6.1 & $180^{\prime} 104.87$ \\
\hline $2 \mathrm{~L}$ & Bev + FOLFOX 4 (E3200) & 7.3 & $248^{\prime} 216.37$ & 4.0 & $136^{\prime} 008.97$ \\
\hline $3 \mathrm{~L}$ & BSC - wild-type KRAS (CO.17) & 1.9 & 485.90 & 2.7 & 690.49 \\
\hline Total & & & $554^{\prime} 347.24$ & & $316^{\prime} 804.34$ \\
\hline
\end{tabular}

* Standard treatment duration; PFS: progression-free survival; TML: through multiple lines; Bev: bevacizumab; Cet: cetuximab; BSC: best supportive care

Table 10. Total costs for each treatment regimen - Scenario 4

\begin{tabular}{|c|c|c|c|c|c|}
\hline & Treatment Regimen & $\begin{array}{c}\text { Treatment } \\
\text { months (PFS) }\end{array}$ & Total Cost (R\$) & $\begin{array}{l}\text { Treatment } \\
\text { months* }\end{array}$ & Total Cost (R\$) \\
\hline \multicolumn{6}{|c|}{ Bevacizumab TML } \\
\hline $1 \mathrm{~L}$ & Bev + FOLFIRI (CALGB/SWOG 80405) & 10.8 & $260^{\prime} 620.33$ & 6.1 & $147^{\prime} 202.22$ \\
\hline $2 \mathrm{~L}$ & Bev + FOLFOX6 (ML18147) & 5.7 & 155.706 .86 & 4.0 & $109^{\prime} 267.97$ \\
\hline $3 L$ & Cet + BSC - wild-type KRAS (CO.17) & 3.7 & $59^{\prime} 866.50$ & 2.7 & $44^{\prime} 230.45$ \\
\hline Total & & & $476^{\prime} 193.69$ & & $300^{\prime} 700.65$ \\
\hline \multicolumn{6}{|c|}{ Non-bevacizumab TML } \\
\hline $1 \mathrm{~L}$ & Cet + FOLFIRI (CALGB/SWOG 80405) & 10.4 & $305^{\prime} 644.96$ & 6.1 & $180^{\prime} 104.87$ \\
\hline $2 \mathrm{~L}$ & Bev + FOLFOX 4 (E3200) & 7.3 & $248^{\prime} 216.37$ & 4.0 & $136^{\prime} 008.97$ \\
\hline $3 \mathrm{~L}$ & BSC - wild-type KRAS (CO.17) & 1.9 & 485.90 & 2.7 & 690.49 \\
\hline Total & & & $554^{\prime} 347.24$ & & $316^{\prime} 804.34$ \\
\hline
\end{tabular}

* Standard treatment duration; PFS: progression-free survival; TML: through multiple lines; Bev: bevacizumab; Cet: cetuximab; BSC: best supportive care 
Table 11. Total costs for each treatment regimen - Scenario 5

\begin{tabular}{|c|c|c|c|c|c|}
\hline & Treatment Regimen & $\begin{array}{c}\text { Treatment } \\
\text { months (PFS) }\end{array}$ & Total Cost (R\$) & $\begin{array}{l}\text { Treatment } \\
\text { months* }\end{array}$ & Total Cost (R\$) \\
\hline \multicolumn{6}{|c|}{ Bevacizumab TML } \\
\hline $1 \mathrm{~L}$ & Bev + FOLFOX4 (NO16966) & 9.4 & $226^{\prime} 411.94$ & 6.1 & $146^{\prime} 926.89$ \\
\hline $2 \mathrm{~L}$ & Bev + simplified FOLFIRI (ML18147) & 5.7 & $137^{\prime} 549.62$ & 4.0 & $96^{\prime} 526.05$ \\
\hline $3 \mathrm{~L}$ & Cet + BSC - wild-type KRAS (CO.17) & 3.7 & $59^{\prime} 866.50$ & 2.7 & $44^{\prime} 230.45$ \\
\hline Total & & & $423^{\prime} 781.87$ & & $287^{\prime} 632.40$ \\
\hline \multicolumn{6}{|c|}{ Non-bevacizumab TML } \\
\hline $1 \mathrm{~L}$ & Bev + FOLFOX4 (NO16966, Saltz, 2008) & 10.4 & $2266^{\prime} 411.94$ & 6.1 & $146^{\prime} 926.89$ \\
\hline $2 \mathrm{~L}$ & Pan + FOLFIRI (Peeters, 2010) & 5.9 & $14^{\prime} 797.16$ & 4.0 & $98^{\prime} 845.53$ \\
\hline $3 \mathrm{~L}$ & BSC - wild-type KRAS (CO.17) & 1.9 & 485.90 & 2.7 & 690.49 \\
\hline Total & & & $372^{\prime} 695.00$ & & $246^{\prime} 462.92$ \\
\hline
\end{tabular}

* Standard treatment duration; PFS: progression-free survival; TML: through multiple lines; Bev: bevacizumab; Cet: cetuximab; Pan: panitumumab; BSC: best supportive care

Table 12. Total costs for each treatment regimen - Scenario 6

\begin{tabular}{|c|c|c|c|c|c|}
\hline & Treatment Regimen & $\begin{array}{c}\text { Treatment } \\
\text { months (PFS) }\end{array}$ & Total Cost (R\$) & $\begin{array}{l}\text { Treatment } \\
\text { months* }\end{array}$ & Total Cost (R\$) \\
\hline \multicolumn{6}{|c|}{ Bevacizumab TML } \\
\hline $1 \mathrm{~L}$ & Bev + FOLFIRI (BICC-C) & 9.9 & $270 \prime 272.94$ & 6.1 & $147^{\prime} 202.22$ \\
\hline $2 \mathrm{~L}$ & Bev + FOLFOX (ML18147) & 5.7 & $155^{\prime} 706.86$ & 4.0 & $109^{\prime} 267.97$ \\
\hline $3 \mathrm{~L}$ & CET + BSC - wild-type KRAS (CO.17) & 3.7 & $59^{\prime} 866.50$ & 2.7 & $44^{\prime} 230.45$ \\
\hline Total & & & $485^{\prime} 846.29$ & & $300^{\prime} 700.65$ \\
\hline \multicolumn{6}{|c|}{ Non-bevacizumab TML } \\
\hline $1 \mathrm{~L}$ & Bev + FOLFOX4 (NO16966) & 9.4 & $2266^{\prime} 411.94$ & 6.1 & $146^{\prime} 926.89$ \\
\hline $2 \mathrm{~L}$ & Pan + FOLFIRI (Peeters, 2010) & 5.9 & $14^{\prime} 797.16$ & 4.0 & $98^{\prime} 845.53$ \\
\hline $3 \mathrm{~L}$ & BSC - wild-type KRAS (CO.17) & 1.9 & 485.90 & 2.7 & 690.49 \\
\hline Total & & & $372^{\prime} 695.00$ & & $246^{\prime} 462.92$ \\
\hline
\end{tabular}

* Standard treatment duration; PFS: progression-free survival; TML: through multiple lines; Bev: bevacizumab; Cet: cetuximab; Pan: panitumumab; BSC: best supportive care

Table 13. Total costs for each treatment regimen - Scenario 7

\begin{tabular}{|c|c|c|c|c|c|}
\hline & Treatment Regimen & $\begin{array}{c}\text { Treatment } \\
\text { months (PFS) }\end{array}$ & Total Cost (R\$) & $\begin{array}{l}\text { Treatment } \\
\text { months* }\end{array}$ & Total Cost (R\$) \\
\hline \multicolumn{6}{|c|}{ Bevacizumab TML } \\
\hline $1 \mathrm{~L}$ & Bev + FOLFOX4 (NO16966) & 9.4 & $226^{\prime} 411.94$ & 6.1 & $146^{\prime} 926.89$ \\
\hline $2 \mathrm{~L}$ & Bev + simplified FOLFIRI (ML18147) & 5.7 & $137^{\prime} 549.62$ & 4.0 & $966^{\prime} 526.05$ \\
\hline $3 \mathrm{~L}$ & Pan + BSC (Van Cutsem, 2007) & 1.9 & $22^{\prime} 642.69$ & 2.7 & $31^{\prime} 176.46$ \\
\hline Total & & & $386^{\prime} 604.25$ & & $275^{\prime} 629.40$ \\
\hline \multicolumn{6}{|c|}{ Non-bevacizumab TML } \\
\hline $1 \mathrm{~L}$ & Bev + FOLFOX4 (NO16966) & 9.4 & $226^{\prime} 411.94$ & 6.1 & $146^{\prime} 926.89$ \\
\hline $2 \mathrm{~L}$ & Pan + FOLFIRI (Peeters, 2010) & 5.9 & $14^{\prime} 797.16$ & 4.0 & $98^{\prime} 845.53$ \\
\hline $3 \mathrm{~L}$ & BSC - wild-type KRAS (CO.17) & 1.9 & 485.90 & 2.7 & 690.49 \\
\hline Total & & & $372^{\prime} 695.00$ & & $246^{\prime} 462.92$ \\
\hline
\end{tabular}

* Standard treatment duration; PFS: progression-free survival; TML: through multiple lines; Bev: bevacizumab; Cet: cetuximab; Pan: panitumumab; BSC: best supportive care 
Table 14. Total costs for each treatment regimen - Scenario 8

\begin{tabular}{|c|c|c|c|c|c|}
\hline & Treatment Regimen & $\begin{array}{c}\text { Treatment } \\
\text { months (PFS) }\end{array}$ & Total Cost (R\$) & $\begin{array}{l}\text { Treatment } \\
\text { months* }\end{array}$ & Total Cost (R\$) \\
\hline \multicolumn{6}{|c|}{ Bevacizumab TML } \\
\hline $1 \mathrm{~L}$ & Bev 5 mg + FOLFIRI (BICC-C) & 11.2 & $270^{\prime} 272.94$ & 6.1 & $147^{\prime} 202.22$ \\
\hline $2 \mathrm{~L}$ & Bev 5 mg + FOLFOX (ML18147) & 5.7 & $155^{\prime} 706.86$ & 4.0 & $109^{\prime} 267.97$ \\
\hline $3 \mathrm{~L}$ & Pan 6 mg + BSC (Van Cutsem, 2007) & 1.9 & $22^{\prime} 642.69$ & 2.7 & $31^{\prime} 176.46$ \\
\hline Total & & & $448^{\prime} 622.48$ & & $288^{\prime} 646.65$ \\
\hline \multicolumn{6}{|c|}{ Non-bevacizumab TML } \\
\hline $1 \mathrm{~L}$ & Bev 5 mg + FOLFOX4 (NO16966) & 9.4 & $226^{\prime} 411.94$ & 6.1 & $146^{\prime} 926.89$ \\
\hline $2 \mathrm{~L}$ & Pan 6 mg + FOLFIRI (Peeters, 2010) & 5.9 & $14^{\prime} 797.16$ & 4.0 & $98^{\prime} 845.53$ \\
\hline $3 \mathrm{~L}$ & BSC - wild-type KRAS (CO.17) & 1.9 & 485.90 & 2.7 & 690.49 \\
\hline Total & & & $372^{\prime} 695.00$ & & $246^{\prime} 462.92$ \\
\hline
\end{tabular}

* Standard treatment duration ; PFS: progression-free survival; TML: through multiple lines; Bev: bevacizumab; Cet: cetuximab; Pan: panitumumab; BSC: best supportive care

Table 15. Total costs for each treatment regimen - Scenario 9

\begin{tabular}{|c|c|c|c|c|c|}
\hline & Treatment Regimen & $\begin{array}{c}\text { Treatment } \\
\text { months (PFS) }\end{array}$ & Total Cost (R\$) & $\begin{array}{l}\text { Treatment } \\
\text { months* }\end{array}$ & Total Cost (R\$) \\
\hline \multicolumn{6}{|c|}{ Bevacizumab TML } \\
\hline $1 \mathrm{~L}$ & Bev + FOLFOX4 (NO16966) & 9.4 & $226^{\prime} 411.94$ & 6.1 & $146^{\prime} 926.89$ \\
\hline $2 \mathrm{~L}$ & Bev + simplified FOLFIRI (ML18147) & 5.7 & $137^{\prime} 549.62$ & 4.0 & $96^{\prime} 526.05$ \\
\hline $3 \mathrm{~L}$ & CET + BSC - wild-type KRAS (CO.17) & 3.7 & $59^{\prime} 866.50$ & 2.7 & $44^{\prime} 230.45$ \\
\hline Total & & & $423^{\prime} 828.06$ & & $287^{\prime} 683.40$ \\
\hline \multicolumn{6}{|c|}{ Non-bevacizumab TML } \\
\hline $1 \mathrm{~L}$ & Bev + FOLFOX4 (NO16966) & 9.4 & $226^{\prime} 411.94$ & 6.1 & $146^{\prime} 926.89$ \\
\hline $2 \mathrm{~L}$ & Cet + IRI (EPIC) & 4.0 & $122^{\prime} 012.96$ & 4.0 & $122^{\prime} 012.96$ \\
\hline $3 \mathrm{~L}$ & BSC - wild-type KRAS (CO.17) & 1.9 & 485.90 & 2.7 & 690.49 \\
\hline Total & & & $348^{\prime} 910.80$ & & $269^{\prime} 630.35$ \\
\hline
\end{tabular}

* Standard treatment duration; PFS: progression-free survival; TML: through multiple lines; Bev: bevacizumab; Cet: cetuximab; Pan: panitumumab; BSC: best supportive care

Table 16. Total costs for each treatment regimen - Scenario 10

\begin{tabular}{|c|c|c|c|c|c|}
\hline & Treatment Regimen & $\begin{array}{c}\text { Treatment } \\
\text { months (PFS) }\end{array}$ & Total Cost (R\$) & $\begin{array}{l}\text { Treatment } \\
\text { months* }\end{array}$ & Total Cost (R\$) \\
\hline \multicolumn{6}{|c|}{ Bevacizumab TML } \\
\hline $1 \mathrm{~L}$ & Bev + FOLFIRI (BICC-C) & 11.2 & $270^{\prime} 272.94$ & 6.1 & $147^{\prime} 202.22$ \\
\hline $2 \mathrm{~L}$ & Bev + FOLFOX (ML18147) & 5.7 & $155^{\prime} 706.86$ & 4.0 & $109^{\prime} 267.97$ \\
\hline $3 \mathrm{~L}$ & CET + BSC - wild-type KRAS (CO.17) & 3.7 & $59^{\prime} 866.50$ & 2.7 & $44^{\prime} 230.45$ \\
\hline Total & & & $485^{\prime} 846.29$ & & $300^{\prime} 700.65$ \\
\hline \multicolumn{6}{|c|}{ Non-bevacizumab TML } \\
\hline $1 \mathrm{~L}$ & Bev + FOLFOX4 (NO16966) & 9.4 & $226^{\prime} 411.94$ & 6.1 & $146^{\prime} 926.89$ \\
\hline $2 \mathrm{~L}$ & Cet + IRI (EPIC) & 4.0 & $122^{\prime} 012.96$ & 4.0 & $122^{\prime} 012.96$ \\
\hline $3 \mathrm{~L}$ & BSC - wild-type KRAS (CO.17) & 1.9 & 485.90 & 2.7 & 690.49 \\
\hline Total & & & $348^{\prime} 910.80$ & & $269^{\prime} 630.35$ \\
\hline
\end{tabular}

* Standard treatment duration ; PFS: progression-free survival; TML: through multiple lines; Bev: bevacizumab; Cet: cetuximab; BSC: best supportive care 
Table 17. Total costs for each treatment regimen - Scenario 11

\begin{tabular}{|c|c|c|c|c|c|}
\hline & Treatment Regimen & $\begin{array}{c}\text { Treatment } \\
\text { months (PFS) }\end{array}$ & Total Cost (R\$) & $\begin{array}{l}\text { Treatment } \\
\text { months* }\end{array}$ & Total Cost (R\$) \\
\hline \multicolumn{6}{|c|}{ Bevacizumab TML } \\
\hline $1 \mathrm{~L}$ & Bev + FOLFOX4 (NO16966) & 9.4 & $226^{\prime} 411.94$ & 6.1 & $146^{\prime} 926.89$ \\
\hline $2 \mathrm{~L}$ & Bev + simplified FOLFIRI (ML18147) & 5.7 & $137^{\prime} 549.62$ & 4.0 & $96^{\prime} 526.05$ \\
\hline $3 \mathrm{~L}$ & Pan + BSC (Van Cutsem, 2007) & 1.9 & $22^{\prime} 624.69$ & 2.7 & $32^{\prime} 176.46$ \\
\hline Total & & & $386^{\prime} 604.25$ & & $275^{\prime} 629.40$ \\
\hline \multicolumn{6}{|c|}{ Non-bevacizumab TML } \\
\hline $1 \mathrm{~L}$ & Bev + FOLFOX4 (NO16966) & 9.4 & $2266^{\prime} 411.94$ & 6.1 & $146^{\prime} 926.89$ \\
\hline $2 \mathrm{~L}$ & Cet $+\mid \mathrm{IRI}(\mathrm{EPIC})$ & 4.0 & $122^{\prime} 012.96$ & 4.0 & $122^{\prime} 012.96$ \\
\hline $3 \mathrm{~L}$ & BSC - wild-type KRAS (CO.17) & 1.9 & 485.90 & 2.7 & 690.49 \\
\hline Total & & & $348^{\prime} 910.80$ & & $269^{\prime} 630.35$ \\
\hline
\end{tabular}

* Standard treatment duration; PFS: progression-free survival; TML: through multiple lines; Bev: bevacizumab; Cet: cetuximab; Pan: panitumumab; BSC: best supportive care

Table 18. Total costs for each treatment regimen - Scenario 12

\begin{tabular}{|c|c|c|c|c|c|}
\hline & Treatment Regimen & $\begin{array}{c}\text { Treatment } \\
\text { months (PFS) }\end{array}$ & Total Cost (R\$) & $\begin{array}{l}\text { Treatment } \\
\text { months* }\end{array}$ & Total Cost (R\$) \\
\hline \multicolumn{6}{|c|}{ Bevacizumab TML } \\
\hline $1 \mathrm{~L}$ & Bev + FOLFIRI (BICC-C) & 11.2 & $270 \prime 272.94$ & 6.1 & $147^{\prime} 202.22$ \\
\hline $2 \mathrm{~L}$ & Bev + FOLFOX (ML18147) & 5.7 & $155^{\prime} 706.86$ & 4.0 & $109^{\prime} 267.97$ \\
\hline $3 \mathrm{~L}$ & Pan + BSC (Van Cutsem, 2007) & 1.9 & $22^{\prime} 624.69$ & 2.7 & $32^{\prime} 176.46$ \\
\hline Total & & & $448^{\prime} 622.48$ & & $288^{\prime} 646.65$ \\
\hline \multicolumn{6}{|c|}{ Non-bevacizumab TML } \\
\hline $1 \mathrm{~L}$ & Bev + FOLFOX4 (NO16966) & 9.4 & $2266^{\prime} 411.94$ & 6.1 & $146^{\prime} 926.89$ \\
\hline $2 \mathrm{~L}$ & Cet + IRI (EPIC) & 4.0 & $122^{\prime} 012.96$ & 4.0 & $122^{\prime} 012.96$ \\
\hline $3 \mathrm{~L}$ & BSC - wild-type KRAS (CO.17) & 1.9 & 485.90 & 2.7 & 690.49 \\
\hline Total & & & $348^{\prime} 910.80$ & & $269^{\prime} 630.35$ \\
\hline
\end{tabular}

* Standard treatment duration ; PFS: progression-free survival; TML: through multiple lines; Bev: bevacizumab; Cet: cetuximab; Pan: panitumumab; BSC: best supportive care

Table 19. Total costs for each treatment regimen - Scenario 13

\begin{tabular}{|c|c|c|c|c|c|}
\hline & Treatment Regimen & $\begin{array}{c}\text { Treatment } \\
\text { months (PFS) }\end{array}$ & Total Cost (R\$) & $\begin{array}{l}\text { Treatment } \\
\text { months* }\end{array}$ & Total Cost (R\$) \\
\hline \multicolumn{6}{|c|}{ Bevacizumab TML } \\
\hline $1 \mathrm{~L}$ & Bev + FOLFOX4 (NO16966) & 9.4 & $226^{\prime} 411.94$ & 6.1 & $146^{\prime} 926.89$ \\
\hline $2 \mathrm{~L}$ & Bev + simplified FOLFIRI (ML18147) & 5.7 & $137^{\prime} 549.62$ & 4.0 & $96^{\prime} 526.05$ \\
\hline $3 \mathrm{~L}$ & Pan + BSC (Van Cutsem, 2007) & 1.9 & $22^{\prime} 624.69$ & 2.7 & $32^{\prime} 176.46$ \\
\hline Total & $386^{\prime} 604.25$ & & $275^{\prime} 629.40$ & & \\
\hline \multicolumn{6}{|c|}{ Non-bevacizumab TML } \\
\hline $1 \mathrm{~L}$ & Cet + FOLFIRI (CRYSTAL) & 9.9 & $291^{\prime} 047.28$ & 6.1 & $180^{\prime} 104.87$ \\
\hline $2 \mathrm{~L}$ & Bev + FOLFOX 4 (E3200) & 7.3 & $248^{\prime} 216.37$ & 4.0 & $136^{\prime} 008.97$ \\
\hline $3 \mathrm{~L}$ & BSC - wild-type KRAS (CO.17) & 1.9 & 485.90 & 2.7 & 690.49 \\
\hline Total & & & $539^{\prime} 749.55$ & & $316^{\prime} 804.34$ \\
\hline
\end{tabular}

* Standard treatment duration; PFS: progression-free survival; TML: through multiple lines; Bev: bevacizumab; Cet: cetuximab; Pan: panitumumab; BSC: best supportive care 
Table 20. Total costs for each treatment regimen - Scenario 14

\begin{tabular}{|c|c|c|c|c|c|}
\hline & Treatment Regimen & $\begin{array}{c}\text { Treatment } \\
\text { months (PFS) }\end{array}$ & Total Cost (R\$) & $\begin{array}{c}\text { Treatment } \\
\text { months* }\end{array}$ & Total Cost (R\$) \\
\hline \multicolumn{6}{|c|}{ Bevacizumab TML } \\
\hline $1 \mathrm{~L}$ & $\mathrm{Bev}+\mathrm{FOLFIRI}(\mathrm{BICC}-\mathrm{C})$ & 11.2 & $270^{\prime} 272.94$ & 6.1 & $147^{\prime} 202.22$ \\
\hline $2 \mathrm{~L}$ & Bev + FOLFOX (ML18147) & 5.7 & $155^{\prime} 706.86$ & 4.0 & $109^{\prime} 267.97$ \\
\hline $3 \mathrm{~L}$ & Pan + BSC (Van Cutsem, 2007) & 1.9 & $22^{\prime} 624.69$ & 2.7 & $32^{\prime} 176.46$ \\
\hline Total & & & $448^{\prime} 622.48$ & & $288^{\prime} 646.65$ \\
\hline \multicolumn{6}{|c|}{ Non-bevacizumab TML } \\
\hline $1 \mathrm{~L}$ & Cet + FOLFIRI (CRYSTAL) & 9.9 & $291^{\prime} 047.28$ & 6.1 & $180^{\prime} 104.87$ \\
\hline $2 \mathrm{~L}$ & Bev + FOLFOX 4 (E3200) & 7.3 & $248^{\prime} 216.37$ & 4.0 & $136^{\prime} 008.97$ \\
\hline $3 \mathrm{~L}$ & BSC - wild-type KRAS (CO.17) & 1.9 & 485.90 & 2.7 & 690.49 \\
\hline Total & & & $539^{\prime} 749.55$ & & $316^{\prime} 804.34$ \\
\hline
\end{tabular}

* Standard treatment duration ; PFS: progression-free survival; TML: through multiple lines; Bev: bevacizumab; Cet: cetuximab; Pan: panitumumab; BSC: best supportive care

Table 21. Total costs for each treatment regimen - Scenario 15

\begin{tabular}{|c|c|c|c|c|c|}
\hline & Treatment Regimen & $\begin{array}{c}\text { Treatment } \\
\text { months (PFS) }\end{array}$ & Total Cost (R\$) & $\begin{array}{l}\text { Treatment } \\
\text { months* }\end{array}$ & Total Cost (R\$) \\
\hline \multicolumn{6}{|c|}{ Bevacizumab TML } \\
\hline $1 \mathrm{~L}$ & Bev + mFOLFOX6 (CALGB/SWOG 80405) & 10.8 & $271^{\prime} 592.65$ & 6.1 & $153^{\prime} 399.55$ \\
\hline $2 \mathrm{~L}$ & Bev + simplified FOLFIRI (ML18147) & 5.7 & $137^{\prime} 549.62$ & 4.0 & $966^{\prime} 526.05$ \\
\hline $3 \mathrm{~L}$ & Pan + BSC (Van Cutsem, 2007) & 1.9 & $22^{\prime} 624.69$ & 2.7 & $32^{\prime} 176.46$ \\
\hline Total & & & $431^{\prime} 784.97$ & & $282^{\prime} 102.06$ \\
\hline \multicolumn{6}{|c|}{ Non-bevacizumab TML } \\
\hline $1 \mathrm{~L}$ & Cet + FOLFIRI (CALGB/SWOG 80405) & 10.4 & $305^{\prime} 644.96$ & 6.1 & $180^{\prime} 104.87$ \\
\hline $2 \mathrm{~L}$ & Bev + FOLFOX 4 (E3200) & 7.3 & $248^{\prime} 216.37$ & 4.0 & $136^{\prime} 008.97$ \\
\hline $3 \mathrm{~L}$ & BSC - wild-type KRAS (CO.17) & 1.9 & 485.90 & 2.7 & 690.49 \\
\hline Total & & & $554^{\prime} 347.24$ & & $316^{\prime} 804.34$ \\
\hline
\end{tabular}

* Standard treatment duration; PFS: progression-free survival; TML: through multiple lines; Bev: bevacizumab; Cet: cetuximab; Pan: panitumumab; BSC: best supportive care

Table 22. Total costs for each treatment regimen - Scenario 16

\begin{tabular}{|c|c|c|c|c|c|}
\hline & Treatment Regimen & $\begin{array}{c}\text { Treatment } \\
\text { months (PFS) }\end{array}$ & Total Cost (R\$) & $\begin{array}{l}\text { Treatment } \\
\text { months* }\end{array}$ & Total Cost (R\$) \\
\hline \multicolumn{6}{|c|}{ Bevacizumab TML } \\
\hline $1 \mathrm{~L}$ & Bev + FOLFIRI (CALGB/SWOG 80405) & 10.8 & $260^{\prime} 620.33$ & 6.1 & $147^{\prime} 202.22$ \\
\hline $2 \mathrm{~L}$ & Bev + FOLFOX (ML18147) & 5.7 & 155.706 .86 & 4.0 & $109^{\prime} 267.97$ \\
\hline $3 \mathrm{~L}$ & Pan + BSC (Van Cutsem, 2007) & 1.9 & $22^{\prime} 624.69$ & 2.7 & $32^{\prime} 176.46$ \\
\hline Total & & & $438^{\prime} 969.88$ & & $288^{\prime} 646.65$ \\
\hline \multicolumn{6}{|c|}{ Non-bevacizumab TML } \\
\hline $1 \mathrm{~L}$ & Cet + FOLFIRI (CALGB/SWOG 80405) & 10.4 & $305^{\prime} 644.96$ & 6.1 & $180^{\prime} 104.87$ \\
\hline $2 \mathrm{~L}$ & Bev + FOLFOX 4 (E3200) & 7.3 & $248^{\prime} 216.37$ & 4.0 & $136^{\prime} 008.97$ \\
\hline $3 \mathrm{~L}$ & BSC - wild-type KRAS (CO.17) & 1.9 & 485.90 & 2.7 & 690.49 \\
\hline Total & & & $554^{\prime} 347.24$ & & $316^{\prime} 804.34$ \\
\hline
\end{tabular}

* Standard treatment duration ; PFS: progression-free survival; TML: through multiple lines; Bev: bevacizumab; Cet: cetuximab; Pan: panitumumab; BSC: best supportive care 
Table 23. Total treatment cost per month based on PFS approach

\begin{tabular}{|c|c|c|c|c|c|c|}
\hline & $\begin{array}{l}\text { Months of } \\
\text { treatment }\end{array}$ & $\begin{array}{l}\text { Total treatment } \\
\text { cost (R\$) }\end{array}$ & $\begin{array}{l}\text { Treatment cost/ } \\
\text { month (R\$) }\end{array}$ & $\begin{array}{l}\text { Months of } \\
\text { treatment }\end{array}$ & $\begin{array}{l}\text { Total treatment } \\
\text { cost (R\$) }\end{array}$ & $\begin{array}{c}\text { Treatment cost } \\
\text { month (R\$) }\end{array}$ \\
\hline & \multicolumn{3}{|c|}{ Bev TML } & \multicolumn{3}{|c|}{ Non-Bev TML } \\
\hline Scenario 1 & 18.8 & $423^{\prime} 828.06$ & $22^{\prime} 544.05$ & 19.1 & $539^{\prime} 749.55$ & $28^{\prime} 259.14$ \\
\hline Scenario 2 & 20.6 & $485^{\prime} 846.29$ & $23^{\prime} 584.77$ & 19.1 & $539^{\prime} 749.55$ & $28^{\prime} 259.14$ \\
\hline Scenario 3 & 20.2 & $469^{\prime} 008.77$ & $23^{\prime} 218.26$ & 19.6 & $554^{\prime} 347.23$ & $28^{\prime} 283.02$ \\
\hline Scenario 4 & 20.2 & $476^{\prime} 193.69$ & $23^{\prime} 573.94$ & 19.6 & $554^{\prime} 347.23$ & $28^{\prime} 283.02$ \\
\hline Scenario 5 & 18.8 & $423^{\prime} 828.06$ & $22^{\prime} 544.05$ & 17.2 & $372^{\prime} 695.00$ & $21^{\prime} 668.31$ \\
\hline Scenario 6 & 20.6 & $485^{\prime} 846.29$ & $23^{\prime} 584.77$ & 17.2 & $372 ' 695.00$ & $21^{\prime} 668.31$ \\
\hline Scenario 7 & 17.0 & $386^{\prime} 604.25$ & $22^{\prime} 741.43$ & 17.2 & $372^{\prime} 695.00$ & $21^{\prime} 668.31$ \\
\hline Scenario 8 & 18.8 & $448^{\prime} 622.48$ & $23^{\prime} 862.90$ & 17.2 & $372^{\prime} 695.00$ & $21^{\prime} 668.31$ \\
\hline Scenario 9 & 18.8 & $423^{\prime} 828.06$ & $22^{\prime} 544.05$ & 15.3 & $348^{\prime} 910.80$ & $22^{\prime} 804.63$ \\
\hline Scenario 10 & 20.6 & $485^{\prime} 846.29$ & $23^{\prime} 584.77$ & 15.3 & $348^{\prime} 910.80$ & $22^{\prime} 804.63$ \\
\hline Scenario 11 & 17.0 & $386^{\prime} 604.25$ & $22^{\prime} 741.43$ & 15.3 & $348^{\prime} 910.80$ & $22^{\prime} 804.63$ \\
\hline Scenario 12 & 18.8 & $448^{\prime} 622.48$ & $23^{\prime} 862.90$ & 15.3 & $348^{\prime} 910.80$ & $22^{\prime} 804.63$ \\
\hline Scenario 13 & 17.0 & $386^{\prime} 604.25$ & $22^{\prime} 741.43$ & 19.1 & $539^{\prime} 749.55$ & $28^{\prime} 259.14$ \\
\hline Scenario 14 & 18.8 & $448^{\prime} 622.48$ & $23^{\prime} 862.90$ & 19.1 & $539^{\prime} 749.55$ & $28^{\prime} 259.14$ \\
\hline Scenario 15 & 18.4 & $431^{\prime} 784.96$ & $23^{\prime} 466.57$ & 19.6 & $554^{\prime} 347.23$ & $28^{\prime} 283.02$ \\
\hline Scenario 16 & 18.4 & $438^{\prime} 969.88$ & $23^{\prime} 857.06$ & 19.6 & $554^{\prime} 347.23$ & $28^{\prime} 283.02$ \\
\hline
\end{tabular}

Table 24. Total treatment cost per month based on average duration approach

\begin{tabular}{|c|c|c|c|c|c|c|}
\hline & $\begin{array}{l}\text { Months of } \\
\text { treatment }\end{array}$ & $\begin{array}{l}\text { Total treatment } \\
\text { cost (R\$) }\end{array}$ & $\begin{array}{l}\text { Treatment cost/ } \\
\text { month (R\$) }\end{array}$ & $\begin{array}{l}\text { Months of } \\
\text { treatment }\end{array}$ & $\begin{array}{l}\text { Total treatment } \\
\text { cost (R\$) }\end{array}$ & $\begin{array}{l}\text { Treatment cost/ } \\
\text { month (R\$) }\end{array}$ \\
\hline & & Bev TML & & & Non-Bev TML & \\
\hline Scenario 1 & 12.8 & $287^{\prime} 683.40$ & $22^{\prime} 475.27$ & 12.8 & $316^{\prime} 804.34$ & $24^{\prime} 750.34$ \\
\hline Scenario 2 & 12.8 & $300^{\prime} 700.65$ & $23^{\prime} 492.24$ & 12.8 & $316^{\prime} 804.34$ & $24^{\prime} 750.34$ \\
\hline Scenario 3 & 12.8 & $286^{\prime} 950.29$ & $22^{\prime} 417.99$ & 12.8 & $316^{\prime} 804.34$ & $24^{\prime} 750.34$ \\
\hline Scenario 4 & 12.8 & $293^{\prime} 690.27$ & $22^{\prime} 944.55$ & 12.8 & $316^{\prime} 804.34$ & $24^{\prime} 750.34$ \\
\hline Scenario 5 & 12.8 & $287^{\prime} 683.40$ & $22^{\prime} 475.27$ & 12.8 & $246^{\prime} 462.92$ & $19^{\prime} 254.92$ \\
\hline Scenario 6 & 12.8 & $300^{\prime} 700.65$ & $23^{\prime} 492.24$ & 12.8 & $246^{\prime} 462.92$ & $19^{\prime} 254.92$ \\
\hline Scenario 7 & 12.8 & $275^{\prime} 629.40$ & $21^{\prime} 533.55$ & 12.8 & $246^{\prime} 462.92$ & $19^{\prime} 254.92$ \\
\hline Scenario 8 & 12.8 & $288^{\prime} 646.65$ & $22^{\prime} 550.52$ & 12.8 & $246^{\prime} 462.92$ & $19^{\prime} 254.92$ \\
\hline Scenario 9 & 12.8 & $287^{\prime} 683.40$ & $22^{\prime} 475.27$ & 12.8 & $269^{\prime} 630.35$ & $21^{\prime} 064.87$ \\
\hline Scenario 10 & 12.8 & $300^{\prime} 700.65$ & $23^{\prime} 492.24$ & 12.8 & $269^{\prime} 630.35$ & $21^{\prime} 064.87$ \\
\hline Scenario 11 & 12.8 & $275^{\prime} 629.40$ & $21^{\prime} 533.55$ & 12.8 & $269^{\prime} 630.35$ & $21^{\prime} 064.87$ \\
\hline Scenario 12 & 12.8 & $288^{\prime} 646.65$ & $22^{\prime} 550.52$ & 12.8 & $269^{\prime} 630.35$ & $21^{\prime} 064.87$ \\
\hline Scenario 13 & 12.8 & $275^{\prime} 629.40$ & $21^{\prime} 533.55$ & 12.8 & $316^{\prime} 804.34$ & $24^{\prime} 750.34$ \\
\hline Scenario 14 & 12.8 & $288^{\prime} 646.65$ & $22^{\prime} 550.52$ & 12.8 & $316^{\prime} 804.34$ & $24^{\prime} 750.34$ \\
\hline Scenario 15 & 12.8 & $274^{\prime} 896.30$ & $21^{\prime} 476.27$ & 12.8 & $316^{\prime} 804.34$ & $24^{\prime} 750.34$ \\
\hline Scenario 16 & 12.8 & $281^{\prime} 636.27$ & $22^{\prime} 002.83$ & 12.8 & $316^{\prime} 804.34$ & $24^{\prime} 750.34$ \\
\hline
\end{tabular}


Table 25. Parameters that were varied at the one-way sensitivity analysis

\begin{tabular}{|c|c|c|}
\hline Parameter & $\begin{array}{l}\text { Minimum } \\
\text { value }\end{array}$ & $\begin{array}{l}\text { Maximum } \\
\text { value }\end{array}$ \\
\hline Weight & $60.5 \mathrm{~kg}$ & $73.9 \mathrm{~kg}$ \\
\hline BSA & $1.57 \mathrm{~m}^{2}$ & $1.91 \mathrm{~m}^{2}$ \\
\hline $\begin{array}{l}\text { FOLFOX cost ( } 5 F U \text {, leucovorin, } \\
\text { oxaliplatin cost per mg) }\end{array}$ & $\begin{array}{c}\mathrm{R} \$ / \mathrm{mg} 0.04 \\
0.91,34.43\end{array}$ & $\begin{array}{c}\mathrm{R} \$ / \mathrm{mg} 0.05 \\
1.11,42.08\end{array}$ \\
\hline $\begin{array}{l}\text { FOLFIRI cost ( } 5 \mathrm{FU} \text {, leucovorin, } \\
\text { irinotecan cost per mg) }\end{array}$ & $\begin{array}{c}\mathrm{R} \$ / \mathrm{mg} 0.04 \\
0.91,14.91\end{array}$ & $\begin{array}{c}\mathrm{R} \$ / \mathrm{mg} 0.05 \\
1.11,18.23\end{array}$ \\
\hline Bevacizumab cost per mg & $\mathrm{R} \$ 12.23 / \mathrm{mg}$ & $\mathrm{R} \$ 14.94 / \mathrm{mg}$ \\
\hline Cetuximab cost per mg & $\mathrm{R} \$ 6.94 / \mathrm{mg}$ & $\mathrm{R} \$ 8.48 / \mathrm{mg}$ \\
\hline \multicolumn{3}{|l|}{ Panitumumab cost per mg } \\
\hline BSC cost & $\mathrm{R} \$ 230.61$ & $\mathrm{R} \$ 281.31$ \\
\hline Intravenous administration fee & R\$ 165.94 & $R \$ 202.82$ \\
\hline Infusion (>22h) fee & $\mathrm{R} \$ 226.34$ & $R \$ 276.64$ \\
\hline Average treatment duration & 11.5 months & 14.1 months \\
\hline $1 \mathrm{~L}$ & 5.5 months & 6.7 months \\
\hline $2 \mathrm{~L}$ & 3.6 months & 4.4 months \\
\hline $3 \mathrm{~L}$ & 2.4 months & 3.0 months \\
\hline
\end{tabular}

*FOLFOX: bolus and infusion fluorouracil/ leucovorin + oxaliplatin; FOLFIRI: bolus and infusion fluorouracil/leucovorin + irinotecan; BSC: best supportive care

ne, at 6 weeks, and at 3, 6 and 9 months after the randomization, using EORTC QLQ-30 and Dermatology-Specific Quality of Life (DSQL) Scale. There were no differences in global health functioning $(p=0.164)$ or other EORTC items/subscales at 3 months by treatment arm. However, significant differences were found across the arms in skin symptoms ( $p<.0001)$, limitations in social activities due to a skin condition $(p=0.008)$, and concerns about appearance $(p<.0001)$, as measured by DSQL. Patients randomized to receive Bev reported fewer skin symptoms, social limitations and appearance concerns than patients receiving Cet (alone or in combination with Bev) (Naughton et al., 2013). Based on this data, it is widely accepted that either Bev or Cet might be part of the $1 \mathrm{~L}$ therapy for patients with wtRAS mCRC and it seems reasonable to have a cost-minimization approach to compare at least the costs for $1 \mathrm{~L}$ and $2 \mathrm{~L}$ of treatment. Therefore, comparing the total and monthly treatment costs of Bev and Cet in $1 \mathrm{~L}$ treatment in $\mathrm{MCRC}$, the regimens starting with bevacizumab beyond first-progression (Bev TML) were cost-saving in 50\% of all scenarios and in 100\% of scenarios with non-Bev TML regimens starting with Cet (Non-Bev TML). Despite the switch of Cet to Pan in 3 L biologic drug in Bev TML regimens.

There were no scenarios starting with Pan, since both pivotal trials for Pan in $1 \mathrm{~L}$ and Bev in $2 \mathrm{~L}$ were in combination with FOLFOX, which would not allow simulating the backbone chemotherapy switch after first progression.
Regarding the $3 \mathrm{~L}$ treatment options, it might be possible to add irinotecan to Cet in metastatic colorectal cancer patients who were refractory to treatment to irinotecan in prior lines of therapy. Those findings were demonstrated in a phase II trial, BOND (Cunningham et al., 2004). According to the authors, Cet in combination with irinotecan had significant clinical activity in patients who had been previously treated with this drug. Despite its clinical relevance, this scenario with irinotecan in combination with Cet was not included in the analysis due to the study methodology, which included only phase III trials. As irinotecan cost is not a parameter with high influence in the model according to the sensitive analysis, the addition of irinotecan in $3 \mathrm{~L}$ treatment is expected to not have a significant impact on the final results.

The remaining scenarios which Non-Bev TML regimens were cost-saving all started with Bev, followed by an anti-EGFR (Cet or Pan). However, one of the main reasons for this result is the impossibility of adding a biologic drug in $3 \mathrm{~L}$ to these non-Bev TML regimens.

It is relevant to highlight that diverse chemotherapy regimens in combination with monoclonal antibodies resulted in different outcomes for patients, as specified in Table 1. Thus, it is important to consider this information when selecting the most suitable sequence of treatment both from clinical and pharmacoeconomical perspectives.

The use of molecular therapies in $\mathrm{mCRC}$ features a breakthrough in the treatment of many tumors, especially in $m C R C$. The maintenance of Bev with other lines of chemotherapy can spare or delay the use of other molecular therapy in the $2 \mathrm{~L}$ treatment of $\mathrm{mCRC}$, without causing any harm to the patient. Besides that, the possibility of using Bev in sequential lines allows the potential of adding more treatment line options, using biologic drugs, optimizing health and survival outcomes.

\section{Conclusion}

Even though the sequential use of Bev beyond the progression for mCRC might be expected to be more costly than Non-TML regimens because of the addition of an anti-EGFR biologic drug in $3 \mathrm{~L}$ treatment, the Bev TML regimens were cost-saving in 50\% of the scenarios of Non-Bev TML, especially over those which had started with Cet in $1 \mathrm{~L}$ treatment. Despite the possibility of adding either Cet or Pan as a $3 \mathrm{~L}$ treatment for mCRC, the Bev TML regimens allowed not only the optimization of subsequent treatment lines for mCRC patients, but also the enhancement of the long term disease management, as well as, potentially saving resources in the wt RAS mCRC settings in Brazil. In scenarios favoring non-Bev TML, all of them started with Bev.

All sixteen scenarios showed that starting $\mathrm{mCRC}$ treatment in wtRAS patients with Bev seems to be a suitable choice, balancing economic and clinical benefits. 
This study is the first economic assessment evaluating biological sequences in mCRC under Brazilian Supplementary Healthcare system perspective. With new therapeutic options available and better knowledge regarding biomarkers and targeted therapies in oncology, economic studies become more valuable for decision making and should also be expanded to evaluate not only direct treatment costs, but also the quality of life and indirect costs.

\section{Acknowledgments}

Medical writing. The authors thank Evidências - A Kantar Health Company, for providing services of medical writing in the production of this manuscript.

\section{References}

Bennouna J, Sastre J, Arnold D, Osterlund P, Greil R, Van Cutsem E, et al. Continuation of bevacizumab after first progression in metastatic colorectal cancer (ML18147): a randomised phase 3 trial. Lancet Oncol. 2013;14(1):29-37.

Bokemeyer C, Van Cutsem E, Rougier P, Ciardiello F, Heeger S, Schlichting $M$, et al. Addition of cetuximab to chemotherapy as first-line treatment for KRAS wild-type metastatic colorectal cancer: pooled analysis of the CRYSTAL and OPUS randomised clinical trials. Eur I Cancer. 2012:48(10):1466-75.

Cartwright $\mathrm{TH}$. Treatment decisions after diagnosis of metastatic colorectal cancer. Clin Colorectal Cancer. 2012;11(3):155-66.

Chari RS, Helton WS, Marsh RD. Chemotherapy and regional therapy of hepatic colorectal metastases: expert consensus statement by Bartlett et al. Ann Surg Oncol. 2006;13(10):1293-5.

Colucci G, Gebbia V, Paoletti G, Giuliani F, Caruso M, Gebbia N, et al. Phase III randomized trial of FOLFIRI versus FOLFOX4 in the treatment of advanced colorectal cancer: a multicenter study of the Gruppo Oncologico Dell'Italia Meridionale. J Clin Oncol. 2005;23(22):4866-75.

Cremolini C, Loupakis F, Antoniotti C, Lupi C, Sensi E, Lonardi S,et al. FOLFOXIRI plus bevacizumab versus FOLFIRI plus bevacizumab as firstline treatment of patients with metastatic colorectal cancer: updated overall survival and molecular subgroup analyses of the open-label, phase 3 TRIBE study. The Lancet Oncol. 2015;16(13):1305-1316.

Cunningham D, Humblet Y, Siena S, Khayat D, Bleiberg H, Santoro A, Bets D, Mueser M, Harstrick A, Verslype C, Chau I, Van Cutsem E. Cetuximab monotherapy and cetuximab plus irinotecan in irinotecan-refractory metastatic colorectal cancer. N Engl J Med, 2004; 351:337-45.

Douillard JY, Cunningham D, Roth AD, Navarro M, James RD, Karasek P, et al. Irinotecan combined with fluorouracil compared with fluorouracil alone as first-line treatment for metastatic colorectal cancer: a multicentre randomised trial. Lancet. 2000;355(9209):1041-7.

Fuchs CS, Marshall J, Mitchell E, Wierzbicki R, Ganju V, Jeffery M, et al. Randomized, controlled trial of irinotecan plus infusional, bolus, or oral fluoropyrimidines in first-line treatment of metastatic colorectal cancer: results from the BICC-C Study. J Clin Oncol. 2007;25(30):4779-86.

Giantonio BJ, Catalano PJ, Meropol NJ, O'Dwyer PJ, Mitchell EP, Alberts SR, et al. Bevacizumab in combination with oxaliplatin, fluorouracil, and leucovorin (FOLFOX4) for previously treated metastatic colorectal cancer: results from the Eastern Cooperative Oncology Group Study E3200. J Clin Oncol. 2007;25(12):1539-44.
Glimelius B, Oliveira J. Rectal cancer: ESMO clinical recommendations for diagnosis, treatment and follow-up. Ann Oncol. 2008;19 Suppl 2:ii31-2.

Glimelius B, Oliveira J. Rectal cancer: ESMO clinical recommendations for diagnosis, treatment and follow-up. Ann Oncol. 2009;20 Suppl 4:54-6.

GLOBOCAN 2012. Available at: < http://globocan.iarc.fr/Pages/fact_sheets_ cancer.aspx>

de Gramont A, Figer A, Seymour M, Homerin M, Hmissi A, Cassidy J, et al. Leucovorin and fluorouracil with or without oxaliplatin as first-line treatment in advanced colorectal cancer. J Clin Oncol. 2000;18(16):2938-47.

Guan ZZ, Xu JM, Luo RC, Feng FY, Wang LW, Shen L, et al. Efficacy and safety of bevacizumab plus chemotherapy in Chinese patients with metastatic colorectal cancer: a randomized phase III ARTIST trial. Chin J Cancer. 2011;30(10):682-9.

Heinemann V, von Weikersthal LF, Decker T, Kiani A, Vehling-Kaise U, Al-Batran SE, et al. FOLFIRI plus cetuximab versus FOLFIRI plus bevacizumab as first-line treatment for patients with metastatic colorectal cancer (FIRE-3): a randomised, open-label, phase 3 trial. Lancet Oncol. 2014; 15:1065-1075.

Hurwitz H, Fehrenbacher L, Novotny W, Cartwright T, Hainsworth J, Heim W, et al. Bevacizumab plus irinotecan, fluorouracil, and leucovorin for metastatic colorectal cancer. N Engl J Med. 2004;350(23):2335-42.

Hurwitz HI, Fehrenbacher L, Hainsworth JD, Heim W, Berlin J, Holmgren E, et al. Bevacizumab in combination with fluorouracil and leucovorin: an active regimen for first-line metastatic colorectal cancer. J Clin Oncol. 2005;23(15):3502-8.

Instituto Nacional do Câncer (INCA). Estimativa 2014 - Incidência de Câncer no Brasil. Available at: <http://www.inca.gov.br/estimativa/2014/sintesede-resultados-comentarios.asp $>$.

Jackson NA, Barrueco J, Soufi-Mahjoubi R, Marshall J, Mitchell E, Zhang $X$, et al. Comparing safety and efficacy of first-line irinotecan/ fluoropyrimidine combinations in elderly versus nonelderly patients with metastatic colorectal cancer: findings from the bolus, infusional, or capecitabine with camptostar-celecoxib study. Cancer. 2009;115(12):2617-29.

Karapetis CS, Khambata-Ford S, Jonker DJ, O'Callaghan CJ, Tu D, Tebbutt $N C$, et al. K-ras mutations and benefit from cetuximab in advanced colorectal cancer. N Engl J Med. 2008;359(17):1757-65.

Naughton MJ, Schrag D, Venook AP, Niedzwiecki D, et al, editors. Quality of life (QOL) and toxicity among patients in CALGB 80405. J Clin Oncol. 2013; suppl; abstr 3611.

Nordlinger B, Van Cutsem E, Gruenberger T, Glimelius B, Poston G, Rougier $P$, et al. Combination of surgery and chemotherapy and the role of targeted agents in the treatment of patients with colorectal liver metastases: recommendations from an expert panel. Ann Oncol. 2009;20(6):985-92.

Nordlinger B, Van Cutsem E, Rougier P, Kohne CH, Ychou M, Sobrero A, et al. Does chemotherapy prior to liver resection increase the potential for cure in patients with metastatic colorectal cancer? A report from the European Colorectal Metastases Treatment Group. Eur J Cancer. 2007;43(14):2037-45.

Papamichael D, Audisio R, Horiot JC, Glimelius B, Sastre J, Mitry E, et al. Treatment of the elderly colorectal cancer patient: SIOG expert recommendations. Ann Oncol. 2009;20(1):5-16.

Peeters M, Price TJ, Cervantes A, Sobrero AF, Ducreux M, Hotko Y, André T, et al. Randomized phase III study of panitumumab with fluorouracil, leucovorin, and irinotecan (FOLFIRI) compared with FOLFIRI alone as second-line treatment in patients with metastatic colorectal cancer. J Clin Oncol. 2010;28(31):4706-13. 
Rautenberg T, Siebert U, Arnold D, Bennouna J, Kubicka S, Walzer S, et al. Economic outcomes of sequences which include monoclonal antibodies against vascular endothelial growth factor and/or epidermal growth factor receptor for the treatment of unresectable metastatic colorectal cancer. Journal of medical economics. 2014;17(2):99-110.

Saltz LB, Clarke S, Diaz-Rubio E, Scheithauer W, Figer A, Wong R, et al. Bevacizumab in combination with oxaliplatin-based chemotherapy as first-line therapy in metastatic colorectal cancer: a randomized phase III study. J Clin Oncol. 2008;26(12):2013-9.

Saltz LB, Cox JV, Blanke C, Rosen LS, Fehrenbacher L, Moore MJ, et al. Irinotecan plus fluorouracil and leucovorin for metastatic colorectal cancer. Irinotecan Study Group. N Engl J Med. 2000;343(13):905-14.

Schmoll HJ, Cartwright T, Tabernero J, Nowacki MP, Figer A, Maroun J, et al. Phase III trial of capecitabine plus oxaliplatin as adjuvant therapy for stage III colon cancer: a planned safety analysis in 1,864 patients. J Clin Oncol. 2007;25(1):102-9.

Sobrero AF, Maurel J, Fehrenbacher L, Scheithauer W, Abubakr YA, Lutz MP, et al. EPIC: phase III trial of cetuximab plus irinotecan after fluoropyrimidine and oxaliplatin failure in patients with metastatic colorectal cancer. J Clin Oncol. 2008;26(14):2311-9.

Van Cutsem EJ, Oliveira J. Advanced colorectal cancer: ESMO clinical recommendations for diagnosis, treatment and follow-up. Ann Oncol. 2008;19 Suppl 2:ii33-4.
Van Cutsem E, Oliveira J. Advanced colorectal cancer: ESMO clinical recommendations for diagnosis, treatment and follow-up. Ann Oncol. 2009;20 Suppl 4:61-3.

Van Cutsem E, Peeters M, Siena S, Humblet Y, Hendlisz A, Neyns B, et al. Open-label phase III trial of panitumumab plus best supportive care compared with best supportive care alone in patients with chemotherapy-refractory metastatic colorectal cancer. J Clin Oncol. 2007;25(13):1658-64.

Van Cutsem E, Twelves C, Cassidy J, Allman D, Bajetta E, Boyer M, et al. Oral capecitabine compared with intravenous fluorouracil plus leucovorin in patients with metastatic colorectal cancer: results of a large phase III study. J Clin Oncol. 2001;19(21):4097-106.

Venook A, Donna Niedzwiecki D, Heinz-Josef Lenz A, Innocenti F, al. e, editors. CALGB/SWOG 80405: Phase III trial of irinotecan/5-FU/leucovorin (FOLFIRI) or oxaliplatin/5-FU/leucovorin (mFOLFOX6) with bevacizumab (BV) or cetuximab (CET) for patients (pts) with KRAS wild-type (wt) untreated metastatic adenocarcinoma of the colon or rectum (MCRC). 2014 ASCO Annual Meeting. J Clin Oncol. 2014;suppl; abstr LBA3

Welch S, Spithoff K, Rumble RB, Maroun J. Bevacizumab combined with chemotherapy for patients with advanced colorectal cancer: a systematic review. Ann Oncol. 2010;21(6):1152-62. 